\title{
Waveform Estimation from Approximate Quantum Nondemolition Measurements
}

\author{
Sami Boulebnane $\odot,{ }^{1,2}$ Mischa P. Woods, ${ }^{2}$ and Joseph M. Renes $\odot^{2}$ \\ ${ }^{1}$ Department of Computer Science, University College London, London WC1E 6EA, United Kingdom \\ ${ }^{2}$ Institute for Theoretical Physics, ETH Zurich, 8093 Zurich, Switzerland
}

(Received 7 February 2020; revised 22 March 2021; accepted 7 May 2021; published 29 June 2021)

\begin{abstract}
With the advent of gravitational wave detectors employing squeezed light, quantum waveform estimation-estimating a time-dependent signal by means of a quantum-mechanical probe-is of increasing importance. As is well known, backaction of quantum measurement limits the precision with which the waveform can be estimated, though these limits can, in principle, be overcome by "quantum nondemolition" (QND) measurement setups found in the literature. Strictly speaking, however, their implementation would require infinite energy, as their mathematical description involves Hamiltonians unbounded from below. This raises the question of how well one may approximate nondemolition setups with finite energy or finite-dimensional realizations. Here we consider a finite-dimensional waveform estimation setup based on the "quasi-ideal clock" and show that the estimation errors due to approximating the QND condition decrease slowly, as a power law, with increasing dimension. As a result, we find that approximating QND with this system requires large energy or dimensionality. We argue that this result can be expected to also hold for setups based on truncated oscillators or spin systems.
\end{abstract}

DOI: 10.1103/PhysRevLett.127.010502

The general problem of waveform estimation is to estimate a classical time-dependent signal $x(t)$ by coupling it to a probe system and repeatedly measuring the probe. The difficulty in using quantum probe systems is that measurement causes backaction on the probe, limiting the overall precision of the scheme [1]. This is relevant not only to very small probes, e.g., optomechanical systems [2,3], but also very large, such as Laser Interferometer Gravitational Wave Observatory (LIGO) [4], which has recently begun an observing run employing squeezed light [5] as suggested by Caves [6].

To circumvent these limitations, a specific class of measurements-known as quantum nondemolition (QND) — was identified [7] and explored, particularly with application to gravitational wave detectors (see, e.g., $[8,9]$ and references therein). An observable $\hat{O}(t)$ (regarded in the Heisenberg picture) is quantum nondemolition if $\left[\hat{O}(t), \hat{O}\left(t^{\prime}\right)\right]=0$ for all $t$ and $t^{\prime}$. When this condition only holds at discrete times, the observable is termed stroboscopic, otherwise continuous.

A static observable is a simple case of a QND observable in which $\hat{O}(t)=\hat{O}\left(t^{\prime}\right)$, either for all times (continuous) or periodically spaced times (stroboscopic). A prominent example useful for metrology is the "backaction evading

Published by the American Physical Society under the terms of the Creative Commons Attribution 4.0 International license. Further distribution of this work must maintain attribution to the author(s) and the published article's title, journal citation, and DOI. measurement" of the corotating position quadrature of a harmonic oscillator $[9,10]$. Such measurements have been investigated with microwave and optical probes [11,12]. More recently, building on Koopman's formulation of classical mechanics in Hilbert space [13], Tsang and Caves showed how appropriate coupling of several quantum systems enables one to construct a collection of continuous QND observables that satisfy any desired classical equations of motion [14]. For instance, the center of mass $\frac{1}{2}\left(\hat{q}_{1}+\hat{q}_{2}\right)$ and relative momentum $\hat{p}_{1}-\hat{p}_{2}$ of two uncoupled oscillators, one of mass $m$ and the other of negative mass $-m$, are QND observables of the position and momentum of a classical oscillator [15].

Unfortunately, as in this example, the mathematical description of nonstatic QND observables relies on unphysical Hamiltonians whose implementation would require infinite energy [16]. The mathematical issues are similar to those first raised by Pauli, of whether or not a time observable can exist in quantum theory $[17,18]$. Of course, one need only approximate the QND condition by finitedimensional or finite-energy truncations. For instance, to emulate the negative mass oscillator, one can employ symmetric red and blue sidebands of a carrier frequency [14] or use spin systems [19]. The former have been realized in optomechanical settings [20,21]. The latter have already been applied to magnetometry [22] and position measurement [23]; possible applications to LIGO were recently examined by Khalili and Polzik [24].

The question then becomes how the approximation limits the estimation scheme. These limitations may bear (among others) upon the properties of the waveform $x(t)$ to 
be estimated or the frequency at which one is allowed to perform measurements. It is an interesting question of principle, and potentially of practical relevance in the near future, to determine how stringent these restrictions are for a given dimension or energy constraint. For instance, does the approximate quantum nondemolition setup approach the exact one exponentially fast as a function of dimension and energy or with a slower convergence? One may regard the approximation as especially forgiving in the former case, as only a very modest investment would be needed to obtain excellent performance.

Another possible approximate QND system, which constitutes the main focus of this Letter, involves the quasi-ideal clock of [25], a finite-dimensional approximation of an idealized clock governed by the (unbounded) Hamiltonian $\hat{H}=v \hat{p}$, for some constant velocity $v$. The dynamics of the idealized clock are just pure translation, $\hat{q}(t)=\hat{q}(0)+v t$ and $\hat{p}(t)=\hat{p}(0)$, as for the case of a classical free particle, and so its position records the time. Indeed, the idealized clock can be viewed as an instance of Tsang and Caves's construction, since the center of mass and relative momentum of two free (quantum) particles with opposite masses also satisfy the equations of motion of the classical free particle [with $v=\left(\hat{p}_{1}-\hat{p}_{2}\right) / m$ in this case].

The quasi-ideal clock is a particularly simple QND system, as its free dynamics approximate the idealized case exponentially well in the dimension $d$ [25]. Nevertheless, as we show in this Letter, when subject to repeated measurement for waveform estimation, the quasiideal clock only approximates the idealized nondemolition setting with errors decaying as a power law in the dimension. Namely, backaction limits the minimum achievable measurement precision as well as the minimum detectable signal strength to scale as $d^{-1 / 2}$ and $d^{-1 / 4}$, respectively, which translates into an energy scaling of $E^{-1 / 2}$ and $E^{-1 / 4}$. We also discuss the other options and argue that they may be expected to have a similar power law scaling.

Description of quantum measurement.-For context, we begin by reviewing the general framework of quantum measurement, following [1] (Chap. 5). Given a quantum system prepared in a state $|\Psi\rangle$, consider $n$ sequential measurements corresponding to the observables $\hat{q}_{1}, \ldots, \hat{q}_{n}$. The (non-normalized) postmeasurement state, given that one observed $\tilde{q}_{1}, \ldots, \tilde{q}_{n}$, is given by $\left|\Psi^{\prime}\right\rangle=\hat{\Omega}_{n}\left(\tilde{q}_{n}\right) \cdots \hat{\Omega}_{1}\left(\tilde{q}_{1}\right)|\Psi\rangle$, where $\hat{\Omega}_{j}\left(\tilde{q}_{j}\right)$ is the Kraus operator corresponding to outcome $\tilde{q}_{j}$ of the $j$ th measurement. The joint probability distribution of the outcomes $\tilde{q}_{1}, \ldots, \tilde{q}_{n}$ is obtained from the norm squared of the latter. Usually $\hat{\Omega}_{j}\left(\tilde{q}_{j}\right)$ is constructed by "smearing" the projector onto the eigenspace of $\hat{q}_{j}$ associated with the eigenvalue $\tilde{q}_{j}$, e.g., by a Gaussian. Physically, this corresponds to making an imprecise measurement of $\hat{q}_{j}$.
The quasi-ideal clock.-Consider an odd $d$-dimensional Hilbert space, whose basis elements $|k\rangle$ we label using the integers $\mathbb{Z}_{d}$, ranging from $-(d-1) / 2$ to $(d-1) / 2$. The Hamiltonian of the quasi-ideal clock is simply $\hat{H}_{d}=(2 \pi / \sqrt{d}) \sum_{k \in \mathbb{Z}_{d}} k|k\rangle\langle k|$. The discrete Fourier transform of the energy eigenstates defines the "time eigenstates" $\left|\theta_{j}\right\rangle:=(1 / \sqrt{d}) \sum_{k \in \mathbb{Z}_{d}} e^{-(2 \pi i j k) / d}|k\rangle$, which are eigenvalues of the "time operator" $\hat{T}_{d}:=\sum_{j \in \mathbb{Z}_{d}} j\left|\theta_{j}\right\rangle\left\langle\theta_{j}\right|$.

The time eigenstates have the property that $\left|\theta_{j}\right\rangle$ is transformed to $\left|\theta_{j+1}\right\rangle$ under evolution by time $1 / \sqrt{d}$, meaning the system stroboscopically emulates the idealized case of pure translation [26]. Remarkably, this feature persists for all evolution times, up to an exponentially small error (as a function of the dimension or energy), provided the state is restricted to the quasi-ideal states of [25] (and not necessarily otherwise [27]). Essentially, these states consist of a Gaussian superposition of time eigenstates, with mean energy $E \propto d$ above the ground state and width growing as $d^{\lambda}$ for some $\lambda \in(0,1)$.

In the following, we show that the quasi-ideal clock emulates pure translation not only under free Hamiltonian evolution, but also under repeated weak measurements of $\hat{T}_{d}$. However, this approximation breaks down for measurements that are too strong or too frequent. The underlying intuition is that measurements tend to shrink the wave function, until the latter becomes too narrow for the quasiideal state approximations to hold. As will be shown in detail later, since the width of a quasi-ideal state may only be polynomially (and not exponentially) small in $d$, so will the minimum allowed measurement imprecision.

Consider, now, the quasi-ideal clock coupled to a classical waveform through the time-dependent Hamiltonian $[1+x(t)] \hat{H}_{d}$. We eschew the question of how to engineer such a coupling, as our focus is on inprinciple limits. For the idealized clock, $\hat{q}(t)=\hat{q}(0)+t+\int_{0}^{t} d \tau x(\tau)$, so the waveform can, in principle, be reconstructed from $\tilde{q}(t)$. The finite-dimensional analog of the position operator $\hat{q}$ is the rescaled time operator $\hat{\xi}_{j}:=\hat{T}_{d}\left(t_{j}\right) / \sqrt{d}$, as evolution by time $1 / \sqrt{d}$ advances the clock value by precisely this amount when $x=0$. Hence, setting $t_{j}=j / \sqrt{d}$ for integer $j$, one finds that $\hat{\xi}_{j}-\hat{\xi}_{j-1}$ furnishes an estimate of $\int_{t_{j-1}}^{t_{j}} d \tau[1+x(\tau)]$.

We take the initial state to be a quasi-ideal state of variance $\sigma_{s}^{2} d / 4 \pi$, where $1 / d \ll \sigma_{s}^{2} \ll d$, and the measurement precision to be given by $\sigma_{m}$. The evolution of the clock state between $t_{j-1}$ and $t_{j}$ is given by the unitary $e^{-i \hat{H}_{d} \Delta t_{j} / \sqrt{d}}$, where $\Delta t_{j}=1+\int_{j-1}^{j} d \tau x(\tau / \sqrt{d})$. Equivalently, one may regard this as a measurement of a freely evolving quasi-ideal clock at successive time intervals $\Delta t_{1} / \sqrt{d}, \ldots, \Delta t_{n} / \sqrt{d}$.

Backaction scaling.-It would be desirable to compute the lowest-order moments $\left\langle\tilde{\xi}_{j}\right\rangle$ and $\left\langle\tilde{\xi}_{j} \tilde{\xi}_{l}\right\rangle$, but this is awkward due to periodic boundary conditions. Instead, 
for integers $\ell$ and $m$, investigating $\left\langle e^{2 \pi i \ell \tilde{\xi}_{j} / \sqrt{d}} e^{2 \pi i m \tilde{\xi}_{k} / \sqrt{d}}\right\rangle$ leads to a much more tractable problem. These quantities carry information about both the expected values of the measurements and their correlations. For illustration, the random variable $X \sim \mathcal{N}\left(\mu, \sigma^{2}\right)$ with $\alpha \in \mathbb{R}$, yields $\left\langle e^{i \alpha X}\right\rangle=e^{i \alpha \mu} e^{-(1 / 2) \alpha^{2} \sigma^{2}}$. In general, the phase of $\left\langle e^{2 \pi i \tilde{\xi}_{j} / \sqrt{d}}\right\rangle$ carries information about the expectation of $\tilde{\xi}_{j}$ (provided the latter is symmetrically distributed around its mean), while the modulus carries information about its dispersion.

Let us sketch the result for $\left\langle e^{-\left(2 \pi i \tilde{\xi}_{n} / \sqrt{d}\right)}\right\rangle$, corresponding to $\ell=-1, m=0$, reserving details for the Supplemental Material ([28] Sec. II D 1). The expectation value can be divided into three contributions,

$$
\left\langle e^{-\left(2 \pi i \tilde{\xi}_{n} / \sqrt{d}\right)}\right\rangle=e^{-(2 \pi i / d) \sum_{j=1}^{n} \Delta t_{j}} C_{1} C_{2} C_{3},
$$

where $C_{1}$ depends only on $\sigma_{s}, C_{2}$ only on $\sigma_{m}$, and $C_{3}$ on both $\sigma_{s}$ and $\sigma_{m}$, as well as $\left\{\Delta t_{j}\right\}_{j}$. It turns out that $C_{1}$ behaves as $e^{-\left(\pi \sigma_{s}^{2} / 2 d\right)}$ for large $d$, and $e^{-(2 \pi i / d) \sum_{j=1}^{n} \Delta t_{j}-\left(\pi \sigma_{s}^{2} / 2 d\right)}$ is exactly the classical expectation of $e^{-(2 \pi i X / \sqrt{d})}$ when $X \sim \mathcal{N}\left[(1 / \sqrt{d}) \sum_{j=1}^{n} \Delta t_{j},\left(\sigma_{s}^{2} / 4 \pi\right)\right]$. Meanwhile, the factor $C_{2}$ is essentially $e^{-\left(\pi \sigma_{m}^{2} / 2 d\right)}$, provided $\sigma_{m}^{2} \ll d$. Therefore, it becomes trivial, i.e., 1, when $\sigma_{m} \rightarrow 0$. Finally, $C_{3}$ contains the backaction contributions, as it is generally not trivial for vanishing $\sigma_{m}$. When the $\Delta t_{j}$ are integers, which corresponds to commuting $\hat{T}_{d}$ operators and hence no backaction, one can show that $C_{3}$ is indeed equal to 1 .

It turns out that $C_{3}$ has a simple form related to a random walk. Here we give the general picture; the precise details are spelled out in the Supplemental Material ([28] Sec. II C) for all $\ell$ and $m$. The walk takes place on the discrete ring $\mathbb{Z}_{d}$ and the step size varies according to a roughly Gaussian distribution of zero mean and variance $d / 4 \pi \sigma_{m}^{2}$. Nontrivial contributions to $C_{3}$ occur whenever the walk lands on $(d-1) / 2$. Calling $Z_{j}$ the position at step $j$, we have

$C_{3}=\sum_{z_{1} \in \mathbb{Z}_{d}} P\left(z_{1}\right) \mathbb{E}^{z_{1}} \prod_{j=1}^{n} 1-\left(1-e^{2 \pi i \Delta t_{j}}\right) 1_{Z_{j}=(d-1) / 2}$,

where $\mathbb{E}^{z_{1}}$ denotes the expectation for a walk starting at $z_{1}$ and the distribution $P$ depends on $\sigma_{s}$.

To proceed further, we must specify the $\Delta t_{j}$ [or, equivalently, $x(t)]$. We consider three cases. First, halfinteger $\Delta t_{j}=\frac{1}{2}$. As previously mentioned, the case of integer $\Delta t_{j}$ gives $C_{3}=1$, and so the half-integer choice can heuristically be expected to generate the largest backaction on the system, as this is the "furthest away" (for a comparable spacing of measurements) from the case of no backaction. Now let the number of measurements scale with $d$ as $n=2 t \sqrt{d}$, so that the total measurement time is fixed at $\sum_{j=1}^{n}\left(\Delta t_{j} / \sqrt{d}\right)=t$, independent of $d$. The behavior of $C_{3}$ in terms of the scaling of $\sigma_{m}^{2}$ with respect to $d$ is worked out in detail in the Supplemental Material ([28] Sec. II C 2), but the results can be appreciated from the form of (2). The walk will spend a significant amount of time on the last position when the variance after $n$ steps is the size of the ring: $n\left(d / \sigma_{m}^{2}\right) \gg d^{2}$, which for the given choice of $n$ gives the condition $\sigma_{m}^{2} \ll 1 / \sqrt{d}$. The detailed calculation shows that, in this case, $C_{3}$ is bounded away from 1 by $2 t / \sqrt{d}$, while $C_{3} \approx 1$ up to a deviation exponentially small in $d$ when $\sigma_{m}^{2} \gg 1 / \sqrt{d}$. A numerical simulation of the difference of the two cases is illustrated in Fig. 1.

Combining the scaling behavior of $C_{2}$ and $C_{3}$, it is apparent that one cannot achieve a variance smaller than $1 / \sqrt{d}$ on the measurement of $\tilde{\xi}_{n}$. Because of the form of the Hamiltonian, this is essentially $1 / \sqrt{E}$, for $E$ the mean energy of the quasi-ideal clock. This scaling can be approached by taking $\sigma_{m}^{2} \propto d^{-(1 / 2)+\varepsilon}$, with $\varepsilon>0$ small. If, however, $\sigma_{m}^{2} \ll 1 / \sqrt{d}$, the variance on $\tilde{\xi}_{n}$ is at least $\sqrt{d}$.

These results also hold when the $\Delta t_{j}$ come from a random waveform consisting of white noise with variance $\sigma^{2}: x(t)=\sigma[d W(t) / d t]$, as shown in the Supplemental Material ([28] Sec. II D 3). This is a standard method of benchmarking a statistical estimator; see the Cramér-Rao bound in [35]. Finally, in case the waveform is completely general, it is still possible to show that one may achieve a variance as small as $d^{-(1 / 2)+\varepsilon}$ (for all fixed $\varepsilon>0$ ) on the measurement of $\tilde{\xi}_{n}$, but we have no clear proof that this scaling is optimal.
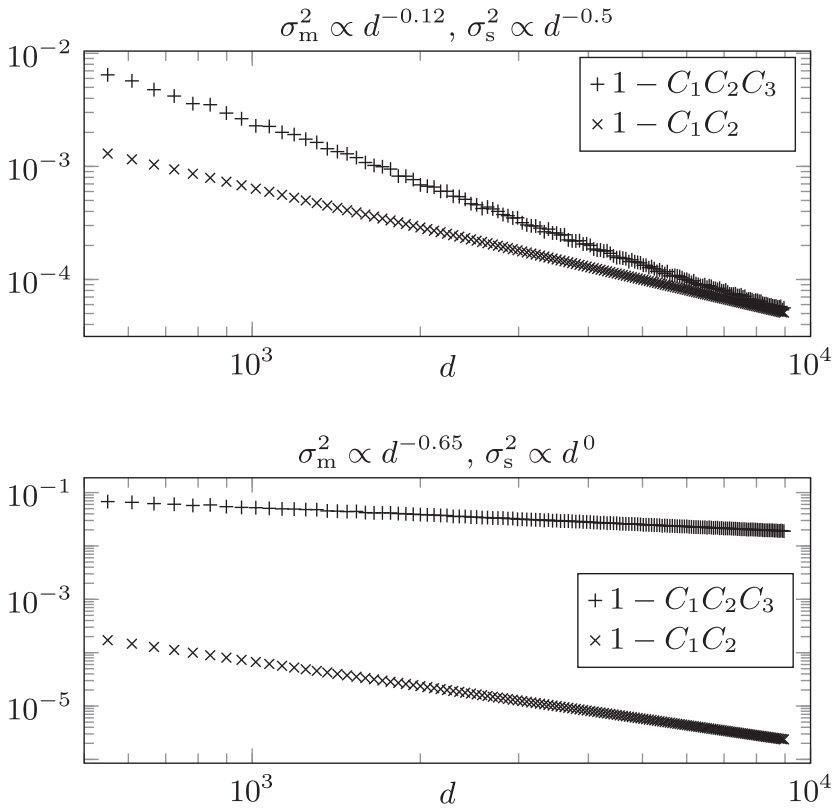

FIG. 1. Scaling of $C_{1}, C_{2}, C_{3}$ with dimension $d$ for different scalings of $\sigma_{s}$ and $\sigma_{m}$. Monte Carlo simulations of 5000 samples, with $t=1$ fixed. 
Waveform estimation.-It remains to be seen whether one can perform efficient waveform estimation given the above constraints on $\tilde{\xi}_{j}$. Returning to the case of continuous $x$, given that typical errors of $\tilde{\xi}_{j}-\tilde{\xi}_{j-1}$ will be roughly of size $d^{-(1 / 4)}$ and from this quantity we aim to estimate $\int_{(j-1) / \sqrt{d}}^{j / \sqrt{d}} d \tau[1+x(\tau)]$, the time average $\langle x\rangle$ of the waveform on a $1 / \sqrt{d}$ time interval must satisfy $|\langle x\rangle| 1 / \sqrt{d} \gg d^{-1 / 4}$ for the error not to overwhelm the expectation. This is unsatisfying, as it means the smallest detectable signal strength increases with increasing dimension as $d^{1 / 4}$. The difficulty is that the measurements are too sharp for how frequently they are occurring, and we should either contemplate weaker measurements at the same frequency or less frequent measurements.

Let us consider the latter. Suppose that the number of measurements $n$ in fixed time $t$ scales as $d^{\gamma}$ for $0 \leq \gamma \leq \frac{1}{2}$. The condition on the variance is now $\sigma_{m}^{2} \gg d^{\gamma-1}$, so that the condition on the waveform becomes $|x| \gg d^{(3 / 2) \gamma-(1 / 2)}$. As a result, provided $\gamma<\frac{1}{3}$, the smallest detectable signal is allowed to vanish as $d \rightarrow \infty$, though strictly slower than $d^{-(1 / 2)}$. Of course, measuring less frequently impacts the useful bandwidth of the procedure, and the maximum detectable frequency $f_{\max }$ is bounded by $f_{\max }<d^{\gamma}$. For example, choosing $\gamma=1 / 6$ and working in terms of $E$ gives $|x| \gg E^{-1 / 4}$ and $f_{\max }<E^{1 / 6}$. This power law scaling in the energy is consistent with other waveform estimation schemes, including the protocol described in [36], which uses a Gottesman-Kitaev-Preskill (GKP) state [37] as a displacement sensor in the context of bosonic quantum error correction. The authors establish that, for a GKP state of energy $E$, the minimum achievable resolution for the displacement is $E^{-1 / 2}$.

Improved scalings can be obtained by more sophisticated estimation procedures, but an exponential improvement seems unlikely. For instance, one may estimate the instantaneous magnitude of the waveform using more than two measurement times considered in the original estimator $\sqrt{d}\left(\tilde{\xi}_{j}-\tilde{\xi}_{j-1}\right)$. For the different problem of estimating a classical force exerted on a quantum harmonic oscillator, considered in the Supplemental Material ([28] Sec. I), this would be analogous to using higher-order finite differences to approximate the derivatives of the position. However, this approach does not seem to work here, as the variance of the estimators diverge in the continuum limit and higherorder differences do not improve the variance, except possibly by a constant factor.

Another way of improving the estimator would be to employ smoothing, as formulated in the quantum case by Tsang [38-40], or indeed any other estimation technique designed for continuous signals. To do so requires a formulation of the measurement process in a suitable limit as a continuous-time process. Note that our setup is outside the usual limiting procedure of ever weaker measurements made ever more often [1,41-43]. In contrast, here we call for stronger measurements made ever more often on an ever larger system, for particular scaling of the former two as a function of the latter.

Using our results and techniques from [44] (Secs. 7 and 13), it can be shown that, as $d \rightarrow \infty$, the measurement process converges weakly to a well-defined continuoustime process in certain cases: to a deterministic motion if the measurement is moderately sharp $\left(1 / \sqrt{d} \ll \sigma_{m}^{2} \ll 1\right)$ and to a Cauchy process with drift for sharp measurements $\left(\sigma_{m}=0\right)$. The former case is precisely the behavior we expect for an idealized clock, namely zero error, which reinforces our conclusions above. A different limit procedure is needed to construct continuous-time estimators for finite $d$, but studying the speed of convergence of this limit may be useful in this regard.

Other approximate QND systems.-The oscillators in the QND construction of [14] can be approximated by truncated oscillators or by spin systems via the HolsteinPrimakoff approximation. Both have free evolution that well approximates the idealized case. Indeed, the time evolution of spin coherent states exactly emulates the idealized case, since a rotation of a spin coherent state by $J_{z}$ produces another spin coherent state. The former behaves similar to the quasi-ideal clock in that the free evolution is exponentially good, provided the wave function is taken much wider than $1 / \sqrt{d}$ but much narrower than $\sqrt{d}$, where $d$ is the truncated dimension, and the energy scales as the dimension. As discussed earlier, this constraint limits the measurement imprecision, which may only decrease as a power law in $d$. Though the analysis is more challenging than for the quasi-ideal clock, numerical investigation confirmed this behavior. Specifically, we simulated the double quantum harmonic oscillator system approximated by spin- $j$ systems described in [14]. The initialization (quasi-ideal state) and measurement protocol (Gaussian Kraus operators) were identical to that of the quasi-ideal clock described above. The results shown in Fig. 2 suggest that the optimal scaling for $\sigma_{m}$ lies between $\sigma_{m} \propto j^{-0.4} \sim d^{-0.2}$ and $\sigma_{m} \propto j^{-0.2} \sim d^{-0.1}$, using that the

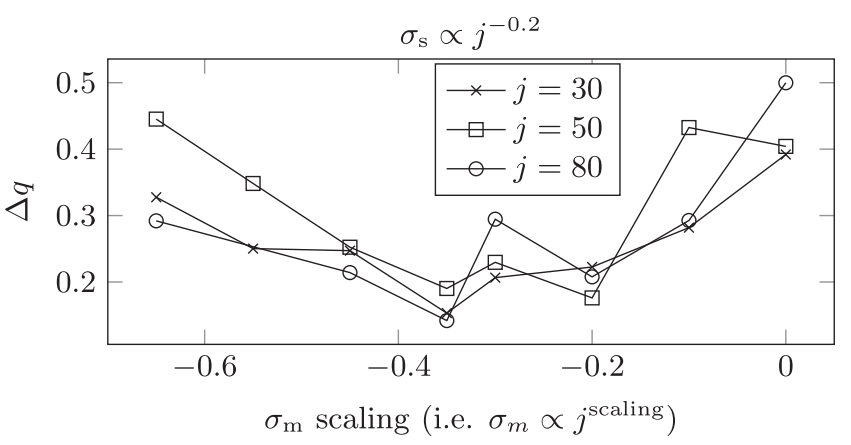

FIG. 2. Standard deviation of position measurement vs measurement imprecision for the double oscillator system simulated by coherent spins. 
total angular momentum number $j$ is related to the dimension $d$ of the emulated system by $d=(2 j+1)^{2}$. Coupled with the fact that two oscillators are needed for the QND setup of [14], it is not unreasonable to expect a worse scaling in estimator accuracy with energy.

Conclusion.-The polynomial scaling of the error in waveform estimation in dimension or energy of the quasiideal clock echoes similar error scalings when it is used for timekeeping [34] or covariant quantum error correction [45]. Indeed, in [45] the bound achieved via the quasi-ideal clock is proven to be the optimally achievable rate permissible by quantum mechanics [46] — suggesting that the scaling derived in this Letter may also be optimal. Its simple structure enables relatively straightforward mathematical analysis, compared with the double oscillator systems; though in light of their practical application $[14,15,24]$, it would be interesting and useful to more thoroughly characterize the error scaling in those cases. To enable a more sophisticated error analysis, it would also be useful to formulate a continuous limit. Perhaps, unlike our considerations above, one can fix $d$ and scale $\sigma_{m}$ and $n$ to obtain a useful limit.

We acknowledge useful discussions with Carlton M. Caves. This work was supported by the Swiss National Science Foundation (SNSF) via the National Centre of Competence in Research "QSIT." M.P.W. acknowledges funding from the SNSF (AMBIZIONE Fellowship No. PZ00P2_179914).

[1] V. B. Braginsky and F. Y. Khalili, Quantum Measurement (Cambridge University Press, Cambridge, England, 1992), https://doi.org/10.1017/CBO9780511622748.

[2] T. J. Kippenberg and K. J. Vahala, Cavity optomechanics: Back-action at the mesoscale, Science 321, 1172 (2008).

[3] J. Cripe, N. Aggarwal, R. Lanza, A. Libson, R. Singh, P. Heu, D. Follman, G. D. Cole, N. Mavalvala, and T. Corbitt, Measurement of quantum back action in the audio band at room temperature, Nature (London) 568, 364 (2019).

[4] LIGO Scientific Collaboration, Enhanced sensitivity of the LIGO gravitational wave detector by using squeezed states of light, Nat. Photonics 7, 613 (2013).

[5] LIGO and Virgo resume search for ripples in space and time, www.ligo.caltech.edu/news/ligo20190326 (2019).

[6] C. M. Caves, Quantum-mechanical noise in an interferometer, Phys. Rev. D 23, 1693 (1981).

[7] V. B. Braginskiı̌ and Y. I. Vorontsov, Quantum-mechanical limitations in macroscopic experiments and modern experimental technique, Sov. Phys. Usp. 17, 644 (1975).

[8] V. B. Braginsky, Y. I. Vorontsov, and K. S. Thorne, Quantum nondemolition measurements, Science 209, 547 (1980).

[9] C. M. Caves, K. S. Thorne, R. W. P. Drever, V. D. Sandberg, and M. Zimmermann, On the measurement of a weak classical force coupled to a quantum-mechanical oscillator. I. Issues of principle, Rev. Mod. Phys. 52, 341 (1980).
[10] K. S. Thorne, R. W. P. Drever, C. M. Caves, M. Zimmermann, and V. D. Sandberg, Quantum Nondemolition Measurements of Harmonic Oscillators, Phys. Rev. Lett. 40, 667 (1978).

[11] J. Suh, A. J. Weinstein, C. U. Lei, E. E. Wollman, S. K. Steinke, P. Meystre, A. A. Clerk, and K. C. Schwab, Mechanically detecting and avoiding the quantum fluctuations of a microwave field, Science 344, 1262 (2014).

[12] I. Shomroni, L. Qiu, D. Malz, A. Nunnenkamp, and T. J. Kippenberg, Optical backaction-evading measurement of a mechanical oscillator, Nat. Commun. 10, 2086 (2019).

[13] B. O. Koopman, Hamiltonian systems and transformation in Hilbert space, Proc. Natl. Acad. Sci. U.S.A. 17, 315 (1931).

[14] M. Tsang and C. M. Caves, Evading Quantum Mechanics: Engineering a Classical Subsystem within a Quantum Environment, Phys. Rev. X 2, 031016 (2012).

[15] M. Tsang and C. M. Caves, Coherent Quantum-Noise Cancellation for Optomechanical Sensors, Phys. Rev. Lett. 105, 123601 (2010).

[16] S. Boulebnane, J. M. Renes, and M. P. Woods, Spectral requirements for quantum nondemolition measurements (to be published).

[17] W. Pauli, Quantentheorie, in Quanten, Handbuch der Physik, edited by W. Bothe, J. Franck, P. Jordan, H. Kulenkampff, R. Ladenburg, W. Noddack, W. Pauli, P. Pringsheim, and H. Geiger (Springer Berlin Heidelberg, Berlin, Heidelberg, 1926), pp. 1-278, https://doi.org/10 .1007/978-3-642-99593-4_1.

[18] E. Galapon, Pauli's theorem and quantum canonical pairs: The consistency of a bounded, self-adjoint time operator canonically conjugate to a Hamiltonian with non-empty point spectrum, Proc. R. Soc. A 458, 451 (2002).

[19] B. Julsgaard, A. Kozhekin, and E. S. Polzik, Experimental long-lived entanglement of two macroscopic objects, Nature (London) 413, 400 (2001).

[20] M. J. Woolley and A. A. Clerk, Two-mode back-actionevading measurements in cavity optomechanics, Phys. Rev. A 87, 063846 (2013).

[21] C. F. Ockeloen-Korppi, E. Damskägg, J.-M. Pirkkalainen, A. A. Clerk, M. J. Woolley, and M. A. Sillanpää, Quantum Backaction Evading Measurement of Collective Mechanical Modes, Phys. Rev. Lett. 117, 140401 (2016).

[22] W. Wasilewski, K. Jensen, H. Krauter, J. J. Renema, M. V. Balabas, and E.S. Polzik, Quantum Noise Limited and Entanglement-Assisted Magnetometry, Phys. Rev. Lett. 104, 133601 (2010).

[23] C. B. Møller, R. A. Thomas, G. Vasilakis, E. Zeuthen, Y. Tsaturyan, M. Balabas, K. Jensen, A. Schliesser, K. Hammerer, and E.S. Polzik, Quantum back-action-evading measurement of motion in a negative mass reference frame, Nature (London) 547, 191 (2017).

[24] F. Y. Khalili and E. S. Polzik, Overcoming the Standard Quantum Limit in Gravitational Wave Detectors Using Spin Systems with a Negative Effective Mass, Phys. Rev. Lett. 121, 031101 (2018).

[25] M. P. Woods, R. Silva, and J. Oppenheim, Autonomous quantum machines and finite-sized clocks, Annales Henri Poincaré 20, 125 (2019).

[26] H. Salecker and E. P. Wigner, Quantum limitations of the measurement of space-time distances, Phys. Rev. 109, 571 (1958). 
[27] A. Peres, Measurement of time by quantum clocks, Am. J. Phys. 48, 552 (1980).

[28] See Supplemental Material at http://link.aps.org/ supplemental/10.1103/PhysRevLett.127.010502 for detailed calculations, which includes Refs. [29-33]. We also include a different proof of the accuracy result of the quasiideal clock as reported in [25,34].

[29] M. Ruzzi, Jacobi theta-functions and discrete Fourier transforms, J. Math. Phys. (N.Y.) 47, 063507 (2006).

[30] P. Diaconis, Group Representations in Probability and Statistics, Lecture Notes-Monograph Series Vol. 11 (Institute of Mathematical Statistics, Hayward, CA, 1988), https://www.jstor.org/stable/4355560.

[31] H. Robbins, A remark on Stirling's formula, Am. Math. Mon. 62, 26 (1955).

[32] C. Saltzer, A brief introduction to theta functions (R. Bellman), SIAM Rev. 5, 162 (1963).

[33] G. E. Andrews, A simple proof of Jacobi's triple product identity, Proc. Natl. Acad. Sci. U.S.A. 16, 333 (1965).

[34] M. P. Woods, R. Silva, G. Pütz, S. Stupar, and R. Renner, Quantum clocks are more accurate than classical ones, arXiv:1806.00491.

[35] H. L. Van Trees, Detection, Estimation, and Modulation Theory, Part I (Wiley, New York, Chichester, 2001), https:// onlinelibrary.wiley.com/doi/book/10.1002/0471221082.

[36] K. Duivenvoorden, B. M. Terhal, and D. Weigand, Single-mode displacement sensor, Phys. Rev. A 95, 012305 (2017).

[37] D. Gottesman, A. Kitaev, and J. Preskill, Encoding a qubit in an oscillator, Phys. Rev. A 64, 012310 (2001).
[38] M. Tsang, Time-Symmetric Quantum Theory of Smoothing, Phys. Rev. Lett. 102, 250403 (2009).

[39] M. Tsang, Optimal waveform estimation for classical and quantum systems via time-symmetric smoothing, Phys. Rev. A 80, 033840 (2009).

[40] M. Tsang, Optimal waveform estimation for classical and quantum systems via time-symmetric smoothing. II. Applications to atomic magnetometry and Hardy's paradox, Phys. Rev. A 81, 013824 (2010).

[41] A. Barchielli, L. Lanz, and G. M. Prosperi, Statistics of continuous trajectories in quantum mechanics: Operationvalued stochastic processes, Found. Phys. 13, 779 (1983).

[42] C. M. Caves and G. J. Milburn, Quantum-mechanical model for continuous position measurements, Phys. Rev. A 36, 5543 (1987).

[43] H. M. Wiseman and G. J. Milburn, Quantum Measurement and Control (Cambridge University Press, Cambridge, England, 2009), https://doi.org/10.1017/ CBO9780511813948.

[44] P. Billingsley, Convergence of Probability Measures, Wiley Series in Probability and Statistics (John Wiley \& Sons, Hoboken, NJ, 1999), https://onlinelibrary.wiley.com/doi/ book/10.1002/9780470316962.

[45] M. P. Woods and Á. M. Alhambra, Continuous groups of transversal gates for quantum error correcting codes from finite clock reference frames, Quantum 4, 245 (2020).

[46] P. Faist, S. Nezami, V. V. Albert, G. Salton, F. Pastawski, P. Hayden, and J. Preskill, Continuous Symmetries and Approximate Quantum Error Correction, Phys. Rev. X 10, 041018 (2020). 